\title{
Rotational Energy Dispersions: Analytic Descriptions
}

\author{
LAWRENCE L. LOHR \\ Department of Chemistry, University of Michigan, Ann Arbor, Michigan 48109-1055
}

\begin{abstract}
Closed-form analytic expressions parametric in the centrifugal displacement are presented for the dependence of the classical rotational energy of a harmonic oscillator (HO), Morse oscillator (MO), and Lennard-Jones 6-12 oscillator (LJO) upon the rotational angular momentum. Powerseries expansions of the energy in terms of the angular momentum are used to construct Padé approximants for the rotational energy dispersion which approximate well the exact parametric solutions and which may be used for fitting experimental spectroscopic data. (c) 1992 Academic Press, Inc.
\end{abstract}

\section{INTRODUCTION}

The distortion of a rotating molecule from its equilibrium geometry and its effect upon energy levels has been recognized for a long time by molecular spectroscopists. Interest in these centrifugal effects, as they are often called, has increased in recent years due to the development of high-resolution spectroscopic techniques and to major advances in the theoretical description of highly excited rotational states of molecules. In a series of recent studies (1-5) we presented a new approach to centrifugal distortions and their associated rotational energy stabilizations which exploits ab initio electronic structure computational methods. This approach is direct, bypassing in the simplest applications the explicit calculation of spectroscopic constants such as vibrational frequencies as this information is implicitly contained in the ab initio electronic energy hypersurface. Specifically the method is particularly useful at any computational level for which analytic gradients of potential-energy hypersurfaces are available. Results were presented in our first study (1) for $\mathrm{H}_{2}^{+}, \mathrm{NH}_{3}, \mathrm{CH}_{4}, \mathrm{BF}_{3}$, and $\mathrm{SF}_{6}$. More detailed studies followed (2-4) of $\mathrm{H}_{2} \mathrm{O}, \mathrm{O}_{3}$, and $\mathrm{PH}_{3}$, as well as an outline of a generalized extension of the method (5). The procedure is structurally oriented, that is, it focuses on the question of the size and shape of molecules with nonzero rotational angular momentum. Centrifugal distortion spectroscopic constants are a very useful form of our computational output, providing an important and indispensable basis for comparison to experimental observations, yet their computation is in a way secondary to the main task. Stated differently, our studies are an exploration of molecular energy in those regions of the nuclear-coordinate hyperspace which are accessible by centrifugal distortions from the equilibrium geometry.

Several questions remain from our ab initio studies. First, what is the best functional form of the rotational encrgy dispersion to use for extrapolating the calculated centrifugal stabilization energies to zero angular momentum and thus for obtaining calculated quartic (and higher) spectroscopic coefficients? Second, why are calculated effective quartic constants approximately linear, rather than quadratic, functions of the angular momentum? Third, why are the decreases in rotational energy associated with centrifugal distortions approximately but not exactly twice the accompanying increases in potential energy? As the answers to these and related questions will also 
provide a foundation for obtaining better expressions to be used in the fitting of experimental spectroscopic data, we present here mathematical analyses of the rotational energy dispersions for classical nonvibrating harmonic ( $\mathrm{HO}$ ), Morse (MO), and Lennard-Jones 6-12 ( $\mathrm{LJO}$ ) oscillators. Insofar as possible, we consider exact relationships for a given model, thus enabling us to distinguish between those features which are a consequence of the choice of a model and those which are a consequence of some approximate description of that model.

\section{ANALYSES}

\section{A. General}

Let the energy of a rotating nonvibrating linear molecule be represented by $E(r$, $J)$, where

$$
E(r, J)=V(r)+\hbar^{2} J(J+1) / 2 m r^{2}
$$

where $V(r)$ is the potential energy for separation $r, m$ is the appropriate reduced mass, and $\hbar^{2} J(J+1)$ is the square of the angular momentum. Note that for reasons of convenience in later applications we use this "quantum" form for the angular momentum even though $J$ here is a continuous rather than a discrete variable. Replacing $r$ by $r_{\mathrm{e}}(1+x)$, where $r_{\mathrm{e}}$ is the equilibrium value of $r$ for $J=0$ and $x$ is a dimensionless displacement variable, defining $B_{\mathrm{e}}$ as $\hbar^{2} / 2 m r_{\mathrm{e}}^{2}$, and dividing by an appropriate reference energy (see below) leads to the dimensionless form

$$
e(x, J)=v(x)+y /(1+x)^{2},
$$

where $y$ is defined by

$$
y=\left(2 B_{\mathrm{e}} / \omega_{\mathrm{e}}\right)^{2} J(J+1)=\alpha^{2} J(J+1) ;
$$

$\omega_{\mathrm{e}}$ is the harmonic vibrational "frequency" in the same units as $B_{\mathrm{e}}$. The quasi-equilibrium separation for a given $y$ ( or $J$ ) is found by setting $d \varepsilon / d x=0$; substitution in Eq. (2) gives an effective reduced energy $\varepsilon_{\mathrm{eff}}(y)$ or $\varepsilon_{\mathrm{eff}}(x)$. Its first form as $\varepsilon_{\mathrm{eff}}(y)$ may be considered as the rotational energy dispersion (energy as a function of angular momentum). While in general this form cannot be found analytically, closed forms can often be found for $\varepsilon_{\mathrm{eff}}(x)$, which, together with closed forms for $y(x)$, comprise analytic parametric representations of the energy dispersion. Closed forms can also often be found for related quantities, such as $d^{2} \varepsilon / d x^{2}$ evaluated at the quasi-equilibrium separations. While this general approach is familiar, many of the results we present below are not.

\section{B. Harmonic Oscillator}

To provide a reference for our studies of the Morse and Lennard-Jones oscillators we first consider the rotating harmonic oscillator (HO). Table I gives the reduced energy $\varepsilon(x)$, the effective energy $\varepsilon_{\mathrm{eff}}(x)$, and the reduced angular momentum squared $y(x)$; the latter two parametrically define the exact dispersion. For the HO we have taken the reference energy to be $k r_{\mathrm{e}}^{2} / 2$, the energy required to compress the HO to $r$ $=0$. We also tabulate the expression for the reduced form of the centrifugal stabilization energy divided by $D_{0}[J(J+1)]^{2}$; this effective quartic constant quantity is $\alpha^{4} \Delta \varepsilon /$ $\delta_{0} y^{2}$ with $\delta_{0}=D_{0} /\left(k r_{\mathrm{e}}^{2} / 2\right), D_{0}=4 B_{\mathrm{e}}^{3} / \omega_{\mathrm{e}}^{2}$, and $\Delta \varepsilon=\varepsilon_{0}-\varepsilon_{\mathrm{eff}}$, with $\varepsilon_{0}$ the reduced rigid rotor energy. Defining $f(y)$ by $\varepsilon_{\mathrm{eff}}(y)=\varepsilon_{0} f(y)$, the quantity $\alpha^{4} \Delta \varepsilon / \delta_{0} y^{2}$ is simply 
TABLE I

Rotational Energy Dispersion Relationships

\begin{tabular}{|c|c|c|}
\hline Oacillator & Quantity & Expressiona \\
\hline \multirow[t]{5}{*}{ HO } & $\varepsilon^{b}$ & $x^{2}+y /(1+x)^{2}$ \\
\hline & $\varepsilon_{\text {eff }}$ & $x(1+2 x)$ \\
\hline & $y^{c}$ & $x(1+x)^{3}$ \\
\hline & $\alpha^{4} \Delta \varepsilon / \delta_{0} y^{2} d$ & {$\left[(1+x)^{2}+x\right] /(1+x)^{6}$} \\
\hline & $E^{n}$ & $(2+8 x) /(1+x)$ \\
\hline \multirow[t]{5}{*}{$\mathrm{MO}^{\mathrm{f}}$} & $\varepsilon$ & {$[1-\exp (-b x)]^{2}+b^{2} y /(1+x)^{2}$} \\
\hline & $\varepsilon_{\text {eff }}$ & {$[1-\exp (-b x)][1+[b(1+x)-1] \exp (-b x)\}$} \\
\hline & $y$ & $\exp (-b x)[1-\exp (-b x)](1+x)^{3 / b}$ \\
\hline & $\alpha^{4} \Delta \varepsilon / \delta_{0} y^{2}$ & $\left.\left\{\exp (-b x)\left[1+b(1+x)\left(x^{2}+2 x\right)\right]-1\right\} / /(1+x)^{6}[1-\exp (-b x)] \exp (-2 b x)\right]$ \\
\hline & $\varepsilon^{\prime \prime}$ & $2 b^{2} \exp (-b x)\{(2 \exp (-b x)+1)+3[(1-\exp (-b x)) b(1+x)]\}$ \\
\hline \multirow[t]{5}{*}{ Lo } & $\varepsilon$ & $z^{3}\left(z^{3}-2\right)+1+36 y z, z \equiv 1 /(1+x)^{2}$ \\
\hline & $\varepsilon_{\text {eff }}$ & $z^{3}\left(-5 z^{3}+4\right)+1$ \\
\hline & $y$ & $\left(1-z^{3}\right) z^{2} / 6$ \\
\hline & $\alpha^{4} \Delta \varepsilon / \delta_{0} y^{2}$ & {$\left[6\left(z^{2}-z^{5}\right)+\left(5 z^{6}-4 z^{3}-1\right) W /\left(1-z^{3}\right)^{2} z^{4}\right.$} \\
\hline & $\varepsilon^{\prime \prime}$ & $24 z^{4}\left(5 z^{3}-2\right)$ \\
\hline \multicolumn{3}{|c|}{$a_{x} \equiv\left(r-r_{e}\right) / r_{e}$} \\
\hline \multicolumn{3}{|c|}{${ }^{b} \varepsilon \equiv E /\left(k r_{e}^{2 / 2)}\right.$ for $H O$ and $E / D_{\theta}$ for $M O$ and $L J O$} \\
\hline \multicolumn{3}{|c|}{$c_{y} \equiv \alpha^{2} J(J+1)$ with $\alpha \equiv\left(2 B_{\Theta} / \omega_{\theta}\right)$} \\
\hline \multicolumn{3}{|c|}{${ }^{d} \delta_{0} \equiv D_{0} /\left(k r_{e}^{2} / 2\right)$ for $H O$ and $D_{0} / D_{e}$ for $M O$ and $L J O, \Delta \varepsilon \equiv \varepsilon_{0}-\varepsilon_{e f f}$. } \\
\hline \multicolumn{3}{|c|}{${ }^{\theta} \varepsilon^{\prime \prime} \equiv\left(d^{2} \varepsilon / d x^{2}\right)$ evaluated for $y=f(x)$} \\
\hline \multicolumn{3}{|c|}{${ }^{f}$ For $M O b=\left(k / 2 D_{e}\right)^{1 / 2} r_{e}$ (dimensionless). } \\
\hline
\end{tabular}

$(1-f) / y$. Although $\varepsilon_{\mathrm{eff}}(y)$ and hence $f(y)$ are not attainable in closed form, we may obtain either by a power-series expansion of $y(x)$, reversion to obtain a series for $x(y)$, and then substitution in $\varepsilon_{\text {eff }}(x)$ to obtain the reduced dispersion $\varepsilon_{\text {eff }}(y)$ and $f(y)$ in the form of a power series. We present in Table II for reference purposes the resulting series for $\varepsilon_{\mathrm{eff}}(y)$ [or $\left.\varepsilon_{\mathrm{eff}}(J)\right]$ with the coefficients $c_{n}$ being integers. Unlike the traditional approaches $(6),{ }^{1}$ no power-series expansion of the potential energy is required.

It is by now well established (7-17) that conventional power-series representations of the dispersion are poorly behaved for large $J$ values (particularly for hydride molecules which have large values of $\alpha$ as defined in Eq. (3)) and that rational function representations, particularly of the Padé form, are more satisfactory. In Tables II and IV we present Padé forms for $\varepsilon_{\text {eff }}(y)$ and $f(y)$, respectively, as constructed from the HO series in Table II to degrees $n=3,4$, and 5. Specifically, from a power series (Table II) for $f(y)$ truncated to degree $n$, where $\varepsilon_{\text {eff }}(y)=\left(\beta_{\mathrm{e}} / \alpha^{2}\right) y f(y)$, we construct (Table III) the Padé approximants $[L / M]=[(n / 2) /(n / 2)]$ for even $n$ and $[((n+$ $1) / 2) /((n-1) / 2)$ ] for odd $n$, where $L$ and $M$ denote the degrees of the highest terms in the numerator and denominator, respectively (18). The Padé approximants in Table IV for $(1-f) / y$ are thus of degrees $[(L-1) / M]$. The coefficients of $y$ in these

${ }^{1}$ The HO expansion coefficients in Table II match the leading term in each Dunham coefficient $Y_{0 j}$, where 0 and $j$ are the powers of $\left(v+\frac{1}{2}\right)$ and $J(J+1)$, respectively. 
TABLE II

Rotational Energy Expansion Coefficients ${ }^{\mathrm{a}}$

\begin{tabular}{ccccc}
\hline$n$ & $c_{n}(H O)$ & \multicolumn{1}{c}{$c_{n}(M O)$} & $c_{n}(L J O)^{c}$ \\
\hline 0 & 1 & 1 & 1 \\
1 & 1 & 1 & 1 \\
2 & 3 & $-b+3$ & 4 \\
3 & 13 & $5 b^{2} / 3-9 b+13$ & $88 / 3$ \\
4 & 68 & $-7 b^{3} / 2+107 b^{2} / 4-72 b+68$ & $832 / 3$ \\
5 & 399 & $42 b^{4} / 5-165 b^{3} / 2+1273 b^{2} / 4-570 b+399$ & 3024 \\
\hline
\end{tabular}

a $H O, M O$, and LJO Oscillator values for $c_{n}$ in $\varepsilon_{e t f}(J)=\beta_{\theta} J(J+1) \sum_{n=0}(-1)^{n} c_{n}\left(\alpha^{2} J(J+1)\right]^{n}$ or $\varepsilon_{e t f}(y)=\left(\beta_{\theta} / \alpha^{2}\right) y f(y)$,

where $\alpha \equiv\left(2 B_{\theta} / \omega_{8}\right)$ (dimensionless), $\beta_{\varepsilon}$ is the (dimensiontess) rotational constant $2 B_{e} / k_{e}{ }^{2}$ for $H O$ and $B_{e} / D_{e}$ for $M O$ and $L J O$, and $y=\alpha^{2} J(J+1)$.

$b_{b} \equiv\left(k / 2 D_{\theta}\right)^{1 / 2 r_{\theta}}$ (dimensionless).

c For LJO, $(-1)^{n}$ in $\varepsilon_{\text {eff }}(J)$ is replaced by -1 for all $n \geq 1$.

rational functions are themselves rational. We compare these various $\mathrm{HO}$ results to their MO and LJO counterparts in a later section.

\section{Morse Oscillator}

Results similar to those for the HO are presented in Tables I-IV for the reduced MO. The reference energy is the dissociation energy $D_{\mathrm{e}}$; the reduced energies $\varepsilon$ and $\varepsilon_{\text {eff }}$, as well as quantities derived from them, contain a dimensionless parameter $b$ equal to $\beta r_{\mathrm{e}}=\left(k / 2 D_{\mathrm{e}}\right)^{1 / 2} r_{\mathrm{e}}$, where $k$ is the curvature of $V(r)$ at $r=r_{\mathrm{e}}$. Some representative $b$ values as calculated from spectroscopic constants $(19)$ are ${ }^{1} \mathrm{H}^{35} \mathrm{Cl}, 2.380$; ${ }^{1} \mathrm{H}^{127} \mathrm{I}, 2.817 ;{ }^{14} \mathrm{~N}_{2}, 2.951$; and ${ }^{127} \mathrm{I}_{2}$, 4.952. The power-series coefficients $c_{n}$ in Table II are polynomials in $b$ with rational coefficients, leading to lengthy Padé expressions for $\varepsilon_{\text {eff }}(y)$ and $f(y)$ in Tables III and IV, respectively. The tables give Padé forms for

TABLE III

Padé Representations ${ }^{a}$ of Rotational Energy Series

\begin{tabular}{|c|c|c|c|c|}
\hline \multirow[t]{2}{*}{ n } & \multirow[t]{2}{*}{ HO } & \multicolumn{2}{|c|}{$M$} & \multirow[t]{2}{*}{ LلJ } \\
\hline & & $b=2$ & $b=6$ & \\
\hline \multirow{2}{*}{3} & $1+10 y / 3-4 y^{2} ; 3$ & $1+2 y / 3-2 y^{2} / 3$ & $1-22 y / 3+10 y^{2} \sqrt{3}$ & $1+25 y / 3+10 y^{2} \pi$ \\
\hline & $1+13 y / 3$ & $1+5 y / 3$ & $1-19 y / 3$ & $1-22 y / 3$ \\
\hline \multirow{2}{*}{4} & $1+25 y / 4+9 y^{2} / 2$ & $1+y-2 y^{2} / 3$ & $1-11 y+18 y^{2}$ & $1-13 y+80 y^{2} / 3$ \\
\hline & $1+20 y / 4+35 y^{2} / 4$ & $1+2 y+y^{2} / 3$ & $\overline{1-10 y+11 y^{2}}$ & $1-12 y+56 y^{2} / 3$ \\
\hline \multirow{2}{*}{5} & $1+278 y / 35+71 y^{2} / 7-79 y^{3} / 35$ & $1+53 y / 10+11 y^{2} / 5-43 y^{3} / 15$ & $1-8171 y / 550+1157 y^{2} / 25-707 y^{3} / 55$ & $1-592 y / 35+415 y^{2} / \pi-274 y^{3} / 21$ \\
\hline & $1+313 y / 35+563 y^{2} / 35$ & $1+63 y / 10+15 y^{2} / 2$ & $1-7621 y / 550+19469 y^{2} / 550$ & $1-557 y / 35+1650 y^{2} / 35$ \\
\hline
\end{tabular}

a Tabulated expressions are consincted from the series in Table II to order $n$ and are for $f(y)$, where $\varepsilon_{e f f}(y)=\left(\beta_{e} / \alpha^{2}\right) y f(y)=e_{0}^{f}(y), y=\alpha^{2} J(J+1)$ and $\alpha=\left(2 B_{\theta} / \omega_{\theta}\right)$. 
TABLE IV

Padé Representations of Relative Centrifugal Stabilization Energies ${ }^{2}$

\begin{tabular}{|c|c|c|c|c|}
\hline \multirow[t]{2}{*}{$\mathbf{n}$} & \multirow[t]{2}{*}{ Hо } & \multicolumn{2}{|c|}{ mob } & \multirow[t]{2}{*}{ LJO } \\
\hline & & $b=2$ & $b=6$ & \\
\hline \multirow{2}{*}{3} & $1+4 y / 3$ & $1+2 y / 3$ & $1-10 y / 3$ & $1-10 y / 3$ \\
\hline & $1+13 y / 3$ & $1+5 y / 3$ & $\overline{1-19 y / 3}$ & $1-22 y / 3$ \\
\hline \multirow[t]{2}{*}{4} & $1+17 y / 4$ & $1+y$ & \multirow{2}{*}{$\frac{1-7 y}{1-10 y+11 y^{2}}$} & $1-8 y$ \\
\hline & $\overline{1+29 y / 4+35 y^{2} / 4}$ & $1+2 y+y^{2} / 3$ & & $\overline{1-12 y+56 y^{2} / 3}$ \\
\hline \multirow{2}{*}{5} & $1+200 y / 35+79 y^{2} / 35$ & $1+53 y / 10+43 y^{2 / 15}$ & $1-5971 y / 550+707 y^{2} / 55$ & $1-417 y / 35+274 y^{2} / 21$ \\
\hline & $1+313 y / 35+563 y^{2} / 35$ & $1+63 y / 10+15 y^{2} / 2$ & $\overline{1-7621 y / 550+19483 y^{2} / 550}$ & $1-557 y / 35+1658 y^{2} / 35$ \\
\hline
\end{tabular}

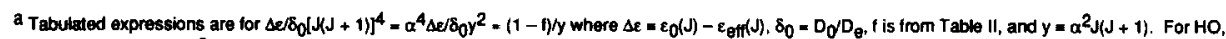
$\mathrm{D}_{\theta}$ is replaced by $\mathrm{kr}_{\theta}{ }^{2 / 2}$.

b The general MO expression for $n=3$ is $\left[1+\left(4-3 b+2 b^{2} / 3\right) y /(3-b)\right] /\left[1+\left(13-9 b+5 b^{2} / 3\right) y /(3-b)\right]$; that for $n=4$ is $\left[1+\left(204-228 b+93 b^{2}-14 b^{3}\right) y /\left(48-36 b+8 b^{2}\right] /\left[1+\left[\left(348-384 b+153 b^{2}-22 b^{3}\right) y+\left(420-600 b+335 b^{2}-87 b^{3}+26 b^{4} / 3\right) y^{2}\right] /\left(48-36 b+8 b^{2}\right)\right]\right.$ while that for $\mathrm{n}=5$ (too lengthy to primt) may be generated from series in $\mathrm{I}$ abe 11 .
}

the special values $b=2$ and $b=6$, while the table footnotes give general expressions as obtained from series of degrees $n=3$ and 4 .

The quantity $\varepsilon_{\text {eff }}$ rises steeply with $x$ and reaches the dissociation limit $\varepsilon_{\text {eff }}=1$ for $x$ values as given in Table $\mathrm{V}$ together with the associated $y$ values (no analytic solution for $x$ or $y$ as a function of $b$ ). Figure 1 shows the dependence of $\varepsilon$ (for $y=0$ ) and $\varepsilon_{\mathrm{eff}}$ on $x$ for $b=2,4$, and 6 . The maximum stable reduced displacement $x$ is typically 0.05 to 0.15 ; larger $x$ and hence $y$ values correspond to predissociating states. Also given in Table $\mathrm{V}$ are numerical values of the curvature of $\varepsilon(x)$ at the quasi-equilibrium for a given $y$; typically this curvature, taken from the expression for $\varepsilon^{\prime \prime}$ in Table $I$, is about one-half of that for $y=0$, meaning that the zero-point energy $(Z P E)$ is a decreasing function of $y$. More specifically, the $y$-dependence of the $Z P E$ may be represented approximately by a series obtained from the square root of the second derivative $\varepsilon^{\prime \prime}$ expression in Table I and the reversion of $y(x)$ to yield $x(y)$; the result is

$$
Z P E(y) / Z P E(0)=1-3(b-1) y / 2+\left(-13 b^{2} / 8+27 b / 4-57 / 8\right) y^{2} \cdots,
$$

TABLE V

Properties $^{\mathrm{a}}$ of $\mathrm{MO}$ with $\varepsilon_{\text {eff }}=1$

\begin{tabular}{lccc}
\hline$b$ & $x$ & $y$ & $\left\langle\varepsilon^{\prime \prime} / \varepsilon_{0}{ }^{\prime \prime}\right)^{b}$ \\
\hline 2 & 0.40323 & 0.34132 & 0.21635 \\
3 & 0.16952 & 0.12782 & 0.32689 \\
4 & 0.08851 & 0.06747 & 0.42751 \\
5 & 0.05341 & 0.04195 & 0.50896 \\
6 & 0.03550 & 0.02869 & 0.57294 \\
\hline \hline
\end{tabular}




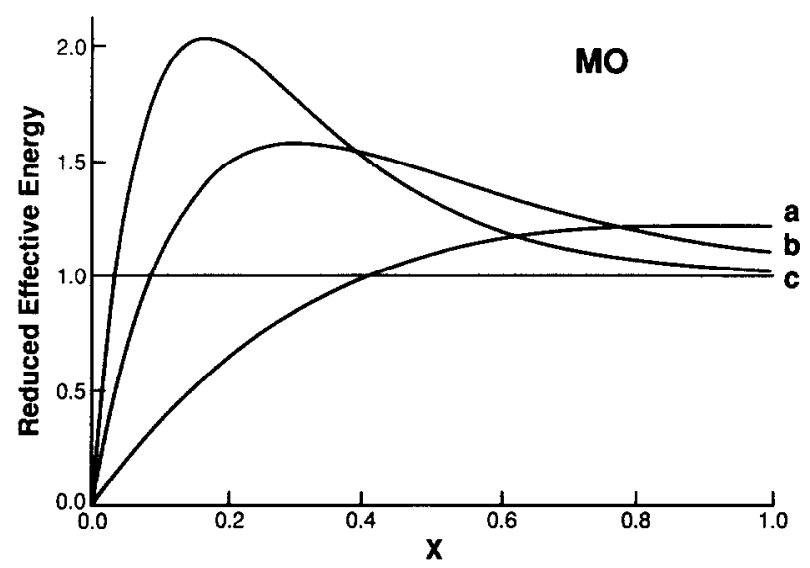

Fig. 1. Plot of MO reduced energy $\varepsilon_{\text {eff }}$ vs reduced displacement $x:$ (a) $b=2$, (b) $b=4$, and (c) $b=6$.

indicating that for $b>1$ the $Z P E$ makes an effective negative contribution to the dispersion $E(J)$. That is, the energy rises less steeply with $J$ than it would if the $Z P E$ were ignored. The leading term in the effect is linear in $y$ and hence linear in $J(J+$ 1 ) and the centrifugal displacement $x$. (Note that the series Eq. (4) is based on a harmonic fit.)

The reduced effective quartic constant previously defined as $\alpha^{4} \Delta \varepsilon / \delta_{0} y^{2}=(1-f) /$ $y$ is shown in Fig. 2 as a function of reduced angular momentum $y^{1 / 2}=\alpha[J(J+$ $1)]^{1 / 2}$ for the MO with $b=2$. The exact form is compared to its power series $(n=$ 4 ) and Padé ([1/2]) approximations. For $b=2$, values of $y^{1 / 2}$ greater than 0.58422 (Table V) correspond to metastable (predissociating) states above the dissociation limit; we note the much better agreement of the Pade form to the exact for $y^{1 / 2}$ values up to this limit. Similar agreement may be seen in plots of the same reduced effective quartic constant vs either $y=\alpha^{2} J(J+1)$ or the displacement $x$.

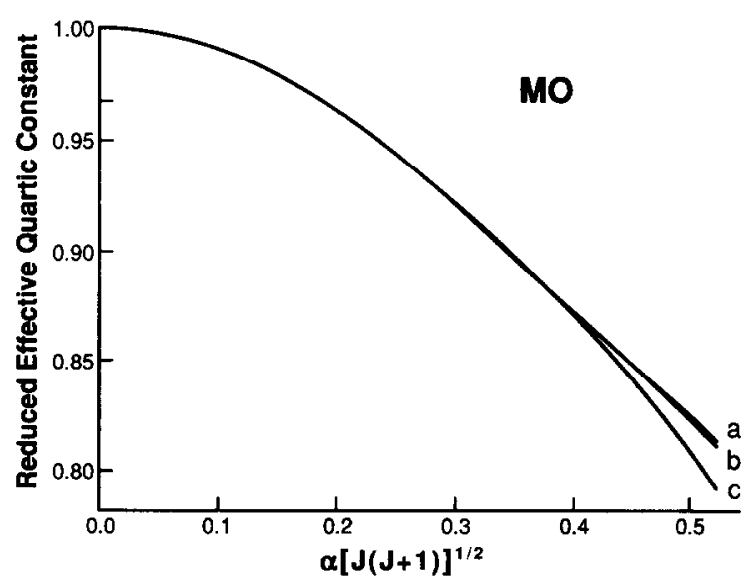

FIG. 2. Plot of MO reduced effective quartic constant $\alpha^{4} \Delta \varepsilon / \delta_{0} y^{2}=(1-f) / y$ vs $y^{1 / 2}=\alpha[J(J+1)]^{1 / 2}$ for $b=2$ : (a) exact, (b) Padé approximant [1/2] from Table III, (c) series approximation to $n=4$. 


\section{Lennard-Jones Oscillator}

Results similar to those for the HO and MO are also presented in Tables I-IV for the reduced 6-12 LJO. Interest in the properties of the LJO has been stimulated by its use as a model for van der Waals molecules. As with the MO, the reference energy is taken as the dissociation energy $D_{\mathrm{e}}$. Many of the expressions for the reduced LJO are conveniently written as polynomials in $z$, where $z$ is $1 /(1+x)^{2}$. As the reduced LJO is completely specified, there are no additional parameters. The series coefficients $c_{n}$ in Table II are rational but not necessarily integral; unlike the HO case where the signs of the terms alternate (the factor $(-1)^{n}$ in the series for $\varepsilon_{\text {eff }}$ ), the LJO terms are all negative for $n \geqslant 1$. That is, all corrections to the rigid-rotor energy are negative for the LJO. The MO case is like the HO case in containing the factor $(-1)^{n}$ in each term, but the sign of the $c_{n}$ 's themselves depend upon the value of $b$.

Table VI gives some special properties of the LJO; the maximum stable centrifugal displacement is only 0.03789 with $y$ being 0.02872 . For ${ }^{40} \mathrm{Ar}_{2}, \alpha=4.642 \times 10^{-3}$ (based (19) on $B_{\mathrm{e}}=0.05975 \mathrm{~cm}^{-1}$ and $\omega_{\mathrm{e}}=25.74 \mathrm{~cm}^{-1}$ ), so that $J_{\max }$ is approximately 36. The barrier to dissociation for this value of $y$ is $\varepsilon_{\max }-1=0.29200$ (in units of $D_{\mathrm{e}}$ ). However, the maximum value for $\varepsilon_{\mathrm{eff}}$ (the largest value for which there is a quasiequilibrium) is much higher, namely $9 / 5$, or $4 / 5$ relative to dissociation, at $x=0.16499$.

Figure 3 shows the dependence of the local LJO energies $\varepsilon(y=0), \varepsilon(y=(4 / 5)$ $2 / 3 / 30$ for which $\varepsilon_{\text {eff }}=1$ ), and $\varepsilon_{\text {eff }}$ on the displacement $x$, while Fig. 4 compares the exact reduced effective quartic constant to its power series $(n=3)$ and Padé $([1 / 1])$ approximations. As with the MO, the Padé form is far superior to the series. Note that this effective LJO quartic constant increases with $J$, and hence with $x$, illustrating the negative energy corrections described above; the MO value decreases unless $b>3$.

We now compare our results to the quantum-mechanical centrifugal constants reported $(20,21)$ for the reduced 6-12 LJO with $D_{\mathrm{e}} / B_{\mathrm{e}}=10^{4}$ (equivalent to specifying the reduced mass), corresponding to $\alpha^{2}=10^{-4} / 36$. From Table II the reduced coefficients (in units of the well depth) through the fifth power of $J(J+1)$ are simply $\delta_{0}$ $=10^{-8} / 36=2.77777 \times 10^{-10}, h=-4 \times 10^{-12} /(36)^{2}=-3.08642 \times 10^{-15}, I=-88$ $\times 10^{-16} / 3(36)^{3}=-6.28717 \times 10^{-20}$, and $m=-832 \times 10^{-20} / 3(36)^{4}=-1.65117$

TABLE VI

Special Properties ${ }^{\text {a }}$ of LJO

\begin{tabular}{llll}
\hline Case & Property & \multicolumn{2}{c}{ Value } \\
\hline$\varepsilon_{\text {eff }}=1$ & $x$ & $(5 / 4)^{1 / 6}-1=0.03789$ \\
& $y$ & $(4 / 5)^{2 / 3} / 30=0.02872$ \\
& $\left(\varepsilon^{\prime \prime} / \varepsilon_{0}\right)^{\prime b}$ & $(4 / 5)^{4 / 3}(2 / 3)=0.49510$ \\
& $\varepsilon_{\max }$ & & \\
& $x\left(\varepsilon_{\max }\right)$ & & 0.51947 \\
& & & \\
$\varepsilon_{\text {eff }}=\max$ & $\varepsilon_{\text {eff }}=$ & $9 / 5$ & \\
& $x$ & $(5 / 2)^{1 / 6}-1=0.16499$ \\
& $y$ & $(2 / 5)^{2 / 3} / 10=0.05429$ \\
\hline
\end{tabular}

a See previous Tables for definitions.

D More generally $2^{4}\left(5 z^{3}-2\right) / 3$; see Table I. 


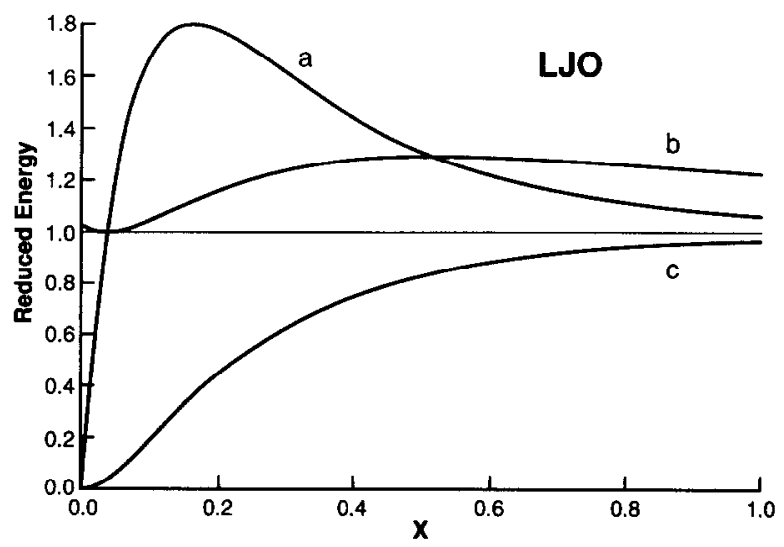

FIG. 3. Plot of LJO reduced energies vs $x$ : (a) $\varepsilon_{\mathrm{eff}}$; (b) $\varepsilon\left(y=(4 / 5)^{2 / 3} / 30\right)$, (c) $\varepsilon(y=0)$.

$\times 10^{-24}$, where the sign of each term corresponds to a negative contribution to the energy. By comparison quantum-mechanical results $(21)$ for $v=0$ are $\delta_{0}=2.93427$ $\times 10^{-10}, h=-3.50081 \times 10^{-15}, I=-7.68573 \times 10^{-20}$, and $m=-2.18223 \times 10^{-24}$. Thus our classical results, based as they are on the assumption of a single effective reduced bond length $1+x$ for a given $J$ and on the neglect of $Z P E$, are qualitatively correct but systematically too small in magnitude, meaning that the energy for a given $J$ is too large. However, the classical dispersion would rise less sharply (the centrifugal constants would increase in magnitude) if we added a $J$-dependent $Z P E$ based on a reduced force constant which may be taken from $\varepsilon^{\prime \prime}$ in Table $I$ as $z^{4}\left(5 z^{3}-2\right) / 3$ times the reduced force constant of 72 for $J=0$, where $z$ again is $1 /(1+x)^{2}$. This expression gives the ratio of $\varepsilon^{\prime \prime} / \varepsilon_{0}^{\prime \prime}=0.49510$ in Table VI for $\varepsilon_{\text {eff }}=1$, meaning that the $Z P E$ decreases by a factor of 0.70363 as the centrifugal displacement approaches $(5 / 4)^{1 / 6}$ -1 . The reduced $Z P E$ for $J=0(y=0)$ is simply $\beta_{\mathrm{e}} / \alpha=6 \beta_{\mathrm{e}}^{1 / 2}$, where $\beta_{\mathrm{e}}=36 \alpha^{2}$ is the ratio of $B_{\mathrm{e}}$ to the well depth, so that $Z P E(z)=6 \beta_{\mathrm{e}}^{1 / 2} z^{2}\left[\left(5 z^{3}-2\right) / 3\right]^{1 / 2}$. The

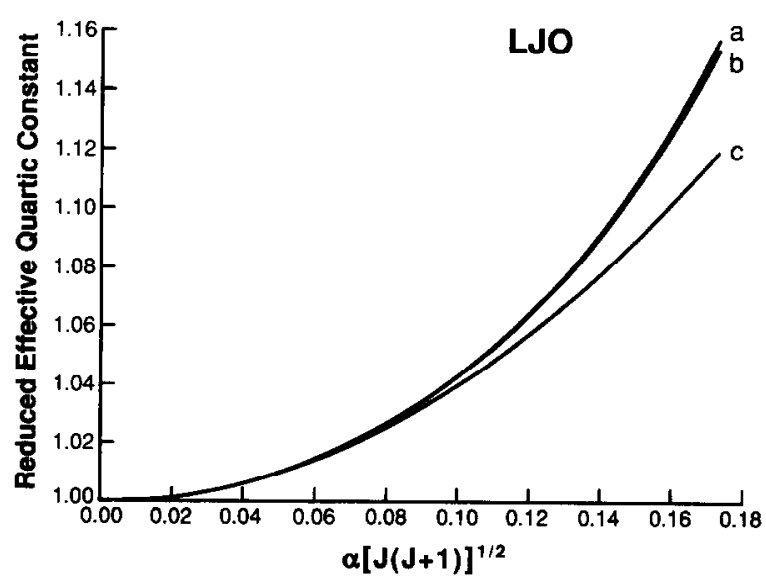

FIG. 4. Plot of LJO reduced effective quartic constant $\alpha^{4} \Delta \varepsilon / \delta_{0} y^{2}=(1-f) / y$ vs $y^{1 / 2}=\alpha[J(J+1)]^{1 / 2}$; (a) exact, (b) Padé approximant [1/1] (Table IV), (c) series approximation to $n=3$. 
LJO $Z P E$ may be obtained as a function of $y$ from the reduced force constant and the series reversion of $y(z)$ or $y(x)$, and is given in units of $6 \beta_{\mathrm{e}}^{1 / 2}$ by

$$
\begin{aligned}
\operatorname{ZPE}(y) / Z P E(0)=1-9 y-65 y^{2} / 2 & -1795 y^{3} / 6 \\
& -28005 y^{4} / 8-1103533 y^{5} / 24+\ldots .
\end{aligned}
$$

For $\beta_{\mathrm{e}}=10^{-4}$, our corrected classical coefficients become $\delta_{0}=2.92824 \times 10^{-10}, h=$ $-3.47115 \times 10^{-15}, I=-7.53768 \times 10^{-20}$, and $m=-2.10743 \times 10^{-24}$, in perhaps surprisingly good agreement with the quantum-mechanical results quoted above.

\section{E. Further Comparisons}

The crucial quantity $\varepsilon_{\text {eff }}$ rises steeply with $x$ because a large fraction of it is rotational energy $\varepsilon_{\mathrm{r}}$. The fraction $\varepsilon_{\mathrm{r}} / \varepsilon_{\mathrm{eff}}$ is given in Table VII for the HO, MO, and LJO. In each case the ratio is 1 in the limit $x=0$ but falls as $x$ increases. Also given in Table VII are expressions for the fraction $\Delta \varepsilon_{\mathrm{r}} / v$, where $\Delta \varepsilon_{\mathrm{r}}$ is $\varepsilon_{0}-\varepsilon_{\mathrm{r}}$, the difference between the energy of the rigid rotor and the rotational energy of the nonrigid rotor ( $\varepsilon_{\mathrm{r}}$ is not $\varepsilon_{\mathrm{eff}}$ ), and $v(x)$ is the potential energy in Eq. (2) of the nonrigid rotor. Expansions of the expressions for this ratio in Table VII give as leading terms $2+3 x$ for the HO, $2+$ $(3-b) x$ for the MO, and $2-4 x$ for the LJO. Thus, both the HO and the MO with $b<3$ give a ratio increasing from 2 as $x$ increases. We note that the coefficient of $x$ matches the expansion coefficient $c_{2}$ in Table II taken together with its sign (note the factor of -1 for the $\mathrm{LJO}$ ) and is thus related to the sextic spectroscopic constant. The leading term of $y(x)$ in Table $\mathrm{I}$ is $x$ for the $\mathrm{HO}, \mathrm{MO}$, and $\mathrm{LJO}$, so that $x$ is proportional to $J(J+1)$ for small $x$ in each case. Thus the ratio $\Delta \varepsilon_{\mathrm{r}} / v$ is simply another measure of the deviation of the rotational energy from a purely quadratic plus quartic dependence. For example, in our calculations for $\mathrm{CH}_{4}$ at the $\mathrm{HF} / 6-31 G^{* *}$ level with $\mathbf{J} \| S_{4}$ we found ( 1 ) this ratio to increase from 2.05 to 2.30 as $J$ increased from 34 to 84 ; the increase is approximately quadratic in $J$ and therefore linear in the displacement. For $\mathrm{BF}_{3}$ at the $\mathrm{HF} / 6-31 G^{*}$ level with $\mathbf{J} \| C_{3}$, we found the ratio to be close to 2, reaching only 2.01 for $J=406$ as the associated displacements of the $F$ atoms are small $(0.03 \AA$ for $J=406)$.

\section{SUMMARY}

We have presented closed-form analytic expressions parametric in the centrifugal displacement for the dependence of the rotational energy of nonvibrating harmonic,

\section{TABLE VII}

Energy Ratios for HO, MO, and LJO

\begin{tabular}{lll}
\hline Aatlo & \multicolumn{1}{c}{ Systom } & \multicolumn{1}{c}{ Expression } \\
\hline$\varepsilon_{r} \varepsilon_{\text {eff }}{ }^{a}$ & HO & $(1+x) /(1+2 x)$ \\
& MO & $b(1+x) \theta^{-b x} /\left\{1+[b(1+x)-1] \theta^{-b x}\right\}$ \\
& LNO & $6 z^{3}\left(1-z^{3}\right) /\left[z^{3}\left(4-5 z^{3}\right)+1\right], z \equiv 1 /(1+x)^{2}$ \\
& & \\
$\Delta E_{T} N b$ & HO & $(1+x)(2+x)$ \\
& MO & $b x(1+x)(2+x) \theta^{-b x} /\left(1-\theta^{-b x}\right)$ \\
& LO & $6 z^{2} /\left(1+z+z^{2}\right)$ \\
\hline
\end{tabular}

$a_{E_{p}}$ is rotational energy.

b $\Delta \varepsilon_{\mathrm{t}} \equiv \varepsilon_{0}-\varepsilon_{\mathrm{T}}$ ( $\varepsilon_{0}$ is rigid rotor energy), $v$ is the potential energy. 
Morse, and 6-12 Lennard-Jones oscillators on the rotational angular momentum, thus complementing quantum-mechanical $(20,21)$ and semiclassical (22) studies. From these expressions we have obtained Padé approximants for this energy and for effective quartic spectroscopic constants, thus providing a basis for selecting Padé forms for the fitting of spectroscopic data. These expressions may also be used in connection with our ab initio procedures (1-5) to obtain spectroscopic constants by the fitting of calculated centrifugal stabilization energies as functions of angular momentum to the appropriate dispersion forms. If desired, the various series and Padé approximant forms may be augmented by $J$-dependent $Z P E$ contributions based on the analytic $\varepsilon^{\prime \prime}$ expressions in Table $I$ and illustrated by the MO and LJO $Z P E$ series in Eqs. (4) and (5). Results for the LJO are of particular interest since the LJO serves as a model for van der Waals complexes. The maximum reduced centrifugal displacement for the $6-12 \mathrm{LJO}$ is only $(5 / 4)^{1 / 6}-1=0.03789$, with larger displacements corresponding to effective energies above the dissociation limit.

RECEIVED: March 5, 1992

\section{REFERENCES}

1. L. L. Lohr AND J.-M. J. Popa, J. Chem. Phys. 84, 4196-4204 (1986).

2. L. L. Lohr and A. J. Helman, J. Compt. Chem. 8, 307-312 (1987).

3. L. L. LoHR, Int. J. Quantum Chem: Quantum Chem. Symp. 21, 407-415 (1987).

4. A. TALeb-Bendiab AND L. L. LOHR, $J$. Mol. Spectrosc. 132, 413-421 (1988).

5. L. L. LOHR, J. Mol. Struct. (THEOCHEM) 199, 265-270 (1989).

6. For example, see J. L. Dunham, Phys. Rev. 41, 721-731 (1932).

7. S. P. Belov, A. V. Burenin, O. L. Polyansky, and S. M. Shapin, J. Mol. Spectrosc. 90, 579-589 (1981).

8. A. V. Burenin, O. L. Polyanskil, and S. M. ShChapin, Opt. Spectrosc. (Engl. Transl.) 53, 395-398 (1982).

9. A. V. Burenin, T. M. Fevral'skikh, E. N. Karyakin, O. L. Polyansky, and S. M. Shapin, $J$. Mol. Spectrosc. 100, 182-192 (1983).

10. A. V. Burenin, O. L. Polyanskil, And S. M. ShChapin, Opt. Spectrosc. (Engl. Transl.) 54, 256-259 (1983).

11. A. V. Burenin and Vi. G. Tyuterey, J. Mol. Spectrosc. 108, 153-154 (1984),

12. A. V. Burenin, T. M. FeVral'Skikh, A. A. Mel'nikov, and S. M. Shapin, J. Mol. Spectrosc. 109, 1-7 (1985)

13. O. L. Polyansky, J. Mol. Spectrosc. 112, 79-87 (1985).

14. J. K. G. Watson, S. C. Foster, A. R. W. McKellar, P, Bernath, T. amano, F. S. Pan, M. W. Crofton, R. S. Altman, And T. OKa, Can. J. Phys. 62, 1875-1885 (1984).

15. S. C. Foster, A. R. W. MCKellar, And J. K. G. Watson, J. Chem. Phys. 85, 664-670 (1986).

16. S. C. Foster, A. R. W. MCKellar, I. R. Peterkin, J. K. G. Watson, F. S. Pan, M. G. Crofton, R. S. Altman, And T. OKA, J. Chem. Phys. 84, 91-99 (1986).

17. J. K. G. WATSON, S. C. Foster, AND A. R. W. MCKellar, Can. J. Phys. 65, 38-46 (1987).

18. B. W. Char, K. O. Geddes, G. H. Gonnet, B. L. Leong, M. B. MonaGan, and S. M. Watt, "Maple V Library Reference Manual,” pp. 50-51, Springer-Verlag, New York, 1991.

19. K. P. HUBer AND G. HeRzBeRG, "Molecular Spectra and Molecular Structure IV. Constants of Diatomic Molecules," Van Nostrand-Reinhold, New York, 1979.

20. J. M. HutSON, J. Phys. B 14, 851-857 (1981).

21. J. Tellinghuisen, J. Mol. Spectrosc. 122, 455-461 (1987).

22. S. M. KRISCHNER AND J. K. G. WATSON, J. Mol. Spectrosc. 47, 234-242 (1973); 51, 321-333 (1974). 